\title{
Pregnancy-associated breast cancer: optimal treatment options
}

This article was published in the following Dove Press journal:

International Journal of Women's Health

4 November 2014

Number of times this article has been viewed

\section{Elyce Cardonick}

Division of Maternal-Fetal Medicine, Department of Obstetrics and Gynecology, Cooper University Hospital, Camden, NJ, USA
Correspondence: Elyce Cardonick Division of Maternal-Fetal Medicine, Department of Obstetrics and Gynecology, Cooper University Hospital, One Cooper Plaza, Dorrance 623, Camden, NJ 08I03, USA

$\mathrm{Tel}+$ I 856342249 |

Fax + I 8563427023

Email cardonick-elyce@cooperhealth.edu

\begin{abstract}
Cancer is diagnosed approximately once per 1,000 pregnancies; most commonly due to the reproductive age of the women, these include breast, cervical, melanoma, thyroid, and Hodgkin's lymphoma diagnoses. As a single diagnosis, breast cancer is the most common cancer diagnosed during pregnancy. Cancer is expected to complicate pregnancy more often due to the trend for women to delay child bearing to later maternal ages. Delayed first birth is itself a risk factor for breast cancer. Termination of pregnancy has not been shown to afford a survival benefit. While protecting the interests of mother and unborn fetus, breast cancer can be safely diagnosed, staged, and treated during pregnancy with good outcomes for both. Some modification of the protocols used for nonpregnant women with suspicious palpable breast masses is required. This article reviews the challenges for physicians in making the diagnosis of breast cancer during pregnancy and upon diagnosis, counseling patients about treatment options. The consequences of diagnostic investigations and cancer treatment for the exposed fetus are also addressed.
\end{abstract}

Keywords: chemotherapy, in utero exposure, gestational

\section{Introduction}

Cancer can complicate pregnancy, especially as more women delay child bearing to later maternal ages. Cancer is diagnosed approximately once per 1,000 pregnancies, with 3,500 cases of various cancers estimated to be diagnosed annually. ${ }^{1}$ Women are delaying pregnancy to older maternal ages, and delayed first birth is itself a risk factor for breast cancer. The ages of patients in the majority of case series reviewed ranged from 26-49 years, with the majority of breast cancers diagnosed at 30-40 years of age. Although early publications recommended termination of pregnancy when cancer was diagnosed, physicians and patients are becoming aware that cancer can be safely managed during pregnancy, with good outcomes for both mother and neonate. Breast cancer, specifically, is the most common malignancy diagnosed during pregnancy, occurring approximately once per 3,000 pregnancies. ${ }^{1}$ Avoiding a delay in diagnosis is vital. This article will detail the diagnostic and staging options as well as treatment choices and consequences during pregnancy.

\section{Diagnosis}

The most common clinical presentation of breast cancer during pregnancy is a painless mass, usually detected by breast self-examination or an examination during an initial prenatal visit. Newly found breast masses should not be attributed to normal physiologic changes during pregnancy or a delay in diagnosis may occur. The axillae should 
be included in examination of the breasts. The protocol to investigate a breast mass and exclude breast cancer in pregnant women is the same as in nonpregnant women, ie, using clinical examination, histology, mammography, and breast ultrasound. Ultrasound is the first method used to evaluate a mass in the pregnant patient to distinguish solid versus cystic lesions. ${ }^{1}$ Features suggestive of malignancy include architectural distortion, an echogenic halo, parallel orientation, lack of posterior enhancement. Benign lesions are usually round or oval shape and have posterior acoustic enhancement, but this may also occur in high-grade tumors, hormone negative tumors, and tumors in young patients. ${ }^{2}$ Therefore, for the purpose of using ultrasound to evaluate breast lesions during pregnancy, distinction should be limited to solid versus cystic masses, with solid lesions undergoing core biopsies. Sensitivity and specificity are not altered by pregnancy. ${ }^{1}$ Biopsies can be safely performed for suspicious masses at any gestational age, but the pathologist should be alerted to the hyperproliferative changes of the breast during pregnancy. Doses of ionizing radiation less than 0.1 Gray (Gy) do not have fetal effects. The fetal radiation exposure from mammography with abdominal shielding is low (0.004 Gy); however, the change in density, increased water, and decreased fat content of the breasts in pregnancy may hinder the mammographic appearance of a mass. Despite these limitations, mammography is still recommended during pregnancy to detect microcalcifications, architectural distortion or focal asymmetry, masses or multifocal disease, but as in nonpregnant women, a negative mammogram in the setting of a palpable mass should not discourage the performance of a biopsy. Mammography has a sensitivity of approximately $86 \%$ during pregnancy. ${ }^{1}$ Core biopsy is the preferred procedure in pregnancy. Fine needle aspiration is unreliable due to hyperproliferative cellularity of the breast during pregnancy and may be more technically difficult to perform due to the engorgement during pregnancy and is therefore discouraged. ${ }^{3,4}$ Amant et al recommend mammography be limited to one oblique view. If a suspicious mass is noted, craniocaudal and mediolateral oblique views of both breasts are performed. ${ }^{3}$ The specificity and sensitivity of breast magnetic resonance imaging (MRI) in pregnant women has not been reported, requires gadolinium, which crosses the placenta with unknown fetal effects, and requires women to be prone on their abdomens for the study. At this time, MRI is not recommended for diagnosis or evaluation of breast cancer in pregnancy. ${ }^{3}$

Even if not attributed to physiologic changes of pregnancy, the palpation of breast masses during pregnancy is difficult and can delay diagnosis. Average diagnostic delays of 2-6 months have been documented for pregnant patients. ${ }^{5,6}$ A delay in diagnosis may increase the risk of nodal involvement (up to $0.9 \%$ with 1 month delay). ${ }^{7}$

\section{Staging}

Only studies which will affect management during the course of the pregnancy should be performed during pregnancy. After a definitive diagnosis, mammography (with abdominal shield) of the unbiopsied breast is recommended to exclude contralateral disease (fetal exposure 0.004 Gy). Because breast cancer in pregnancy is often diagnosed at an advanced stage, comprehensive systemic staging is often necessary. To exclude pulmonary metastasis, chest radiography with abdominal shielding can be carried out safely (0.0001 Gy). ${ }^{4}$ A liver ultrasound is recommended to detect liver metastases. As the incidence of bony metastasis is low in early stage disease $(3 \%-7 \%)$, bone scan can be delayed until postpartum, as results will unlikely affect management during pregnancy. If the patient is symptomatic and metastases are highly suspected, either a skeletal survey via MRI or a modified bone scan can be performed. Modifications for a bone scan in pregnancy include an indwelling catheter to drain radioactive material from the maternal bladder and using $10 \mathrm{mCi}$ rather than $20 \mathrm{mCi} .{ }^{8} \mathrm{MRI}$ of the brain can be performed for patients with central nervous system symptoms. As noted above, while MRI uses magnetic energy rather than ionizing radiation, and is not necessarily harmful to the fetus during pregnancy, breast MRI is not routinely used for breast cancer in pregnancy due to other considerations, such as required maternal prone positioning and required preparation for the study with gadolinium. ${ }^{3}$

\section{Surgery}

Mastectomy under general anesthesia can be performed at any gestational age with minimal risk to the fetus. ${ }^{9,10}$ After 20 weeks, the patient is positioned with left lateral uterine displacement to alleviate aortocaval compression by the growing uterus. An anesthesiologist familiar with the physiologic changes during pregnancy should be present, as there is delayed gastric emptying which can affect intubation. For optimal fetal tolerance of the procedure, hypovolemia and hypoxia should be minimized. Intraoperative fetal monitoring is performed during a procedure at or after 24 weeks gestation, otherwise fetal viability is documented before and after the procedure. Autologous breast reconstruction is delayed for the best cosmetic results to match the unaffected postpartum breast, but expanders/spacers can be placed 
during pregnancy. Lower extremity compression devices are suggested during surgery until the patient is mobile postoperatively to decrease the risks of deep vein thromboses and pulmonary emboli due to the hypercoagulable state in pregnancy.

\section{Surgical choice}

Pregnant patients should have the same discussion as nonpregnant women with breast cancer about the pros and cons of breast conservation surgery. There does not appear to be survival advantage of mastectomy over breast conservation for pregnancy-associated breast cancer (PABC). ${ }^{11}$ After controlling for age, stage, tumor size, race, and hormone receptor status, Rodriguez et al did not report a difference in survival between women treated with modified radical mastectomy compared with breast conservation surgery in pregnancy associated cases. ${ }^{12}$ Patients choosing breast conservation and patients requiring radiation despite mastectomy would defer radiotherapy until the postpartum. The dosage required for breast cancer treatment would result in too high a fetal exposure during any trimester regardless of the location of the uterine fundus. Earlier publications recommended that mastectomy be preferentially performed for patients diagnosed during the first trimester to avoid too long a period of time after completing anthracycline-based chemotherapy in pregnancy before radiation was given postpartum. Recent evidence showing the safety of taxane treatment has given an alternative to this suggestion, as up to four cycles of taxane treatment may be given every 2 weeks to fill that period of time between completing anthracycline-based therapy and postpartum radiation.

Sentinel lymph node biopsy for staging of the regional lymph nodes can be performed safely during pregnancy so that pregnant patients do not necessarily have to undergo a full axillary dissection. ${ }^{13-16}$ The fetal radiation exposure is very low. ${ }^{17}$ The same day approach is preferred in pregnancy, using the radioactive tracer technetium 99 rather than blue dye. Fetal exposure to the radioactivity is minimal and outweighed by the benefits of avoiding unnecessary axillary dissection. Lymphazurin blue dye carries a risk for anaphylaxis, and the use of methylene blue is not recommended in pregnancy due to the fetal risks of methemoglobin, although this was mainly reported when used with amniotic cavity injection.

\section{Histologic type and receptors}

Despite the immunocompromised state of pregnancy, the majority of publications do not find inflammatory breast cancer to be more common in women diagnosed during pregnancy. As in nonpregnant women, the predominant histological type is invasive ductal $(70 \%-90 \%)$, followed by invasive lobular carcinoma. ${ }^{4,6,18}$ Some studies have shown a higher rate of receptor negative cancers in PABC, $, 18,19$ and other studies find receptor and HER2neu status comparable to nonpregnant premenopausal women with breast cancer. ${ }^{20-23}$ Middleton et al found similar rates of HER2neu expression in pregnant and nonpregnant young women with breast cancer. ${ }^{20}$ It appears that age rather than pregnancy determines the biologic features of breast cancer.

\section{Prognosis}

Women diagnosed during pregnancy with breast cancer stages I and IIA have similar survival rates compared to nonpregnant women. ${ }^{6,24-29}$ Age at diagnosis may affect cancer aggressiveness in pregnant woman. ${ }^{30-32}$ Not all studies show similar survival for pregnant women. ${ }^{12,18,19,24-25}$ Johansson et al reported the difference in mortality between PABC and non-PABC was more pronounced among women older than 35 years and among women with PABC diagnosed within 1 year postpartum. ${ }^{32}$

Women with PABC diagnosed at later stages have been reported to have a lower survival. In a retrospective study of women younger than 30 years of age, diagnosed with late stage breast cancer, Anderson et al reported a lower diseasefree and overall survival for PABC compared to controls. ${ }^{33}$ Pregnancy-associated cases also had larger tumors and more advanced stages (more IIIA versus IIB in controls) and more extensive lymph node involvement. Additionally, pregnancy-associated cases included women diagnosed during pregnancy or up to 1 year postpartum. ${ }^{33}$ There was no difference in survival for stages I/IIA. Rodriguez et al reported PABC (defined as diagnosed 9 months before or up to 1 year after delivery) as having more advanced stages of disease and larger primary tumors. ${ }^{12}$ Women with PABC were significantly more likely to have estrogen receptor (ER) negative tumors, a recognized poor prognostic indicator. A 14\% higher death rate was reported for PABC. Women with $\mathrm{PABC}$ have a marginally significantly higher risk of dying $(P=0.046)$ while controlling for stage, tumor size, race, type of surgery, and receptors. ${ }^{12}$ This study included 610 women diagnosed during the postpartum period. In most of these studies, patients were not matched according to treatment protocols, so it is possible that pregnant women were not treated similarly to nonpregnant women, for example, with respect to the delaying of taxane treatment until after delivery. 
Pregnancy was shown in a small study from Norway and one from France to have a worse prognosis after controlling for age, stage at diagnosis, tumor size, lymph node involvement, and date of treatment \pm 1 year. ${ }^{18,34}$ Negative receptor status, larger tumors, and inflammatory cancers were higher in the PABC group. Delay between the first clinical sign of tumor and definitive diagnosis was significantly longer in the PABC group. Survival outcomes were directly related to the delay between the last pregnancy and diagnosis of breast cancer. ${ }^{18}$

The literature consistently notes that women diagnosed within the first 2-3 years after a pregnancy have a worse prognosis, yet the majority of the case control series include women diagnosed up to 1 year postpartum. In a meta-analysis of 30 published studies on breast cancer diagnosed during pregnancy or postpartum, Azim et al concluded the prognosis of breast cancer arising in the postpartum period was significantly associated with poor overall survival compared to patients diagnosed during pregnancy. ${ }^{31}$

Amant et al excluded postpartum cases in this collaborative case-control study of breast cancer in 311 women younger than 45 years. ${ }^{35}$ Follow-up continued to a mean of 61 months. Overall survival was comparable to that of 865 nonpregnant patients matched for age, stage, grade and histologic type of tumor, hormonal receptors, HER2neu status, and type of treatment. There was no statistical difference in disease-free survival, recurrence (hazard ratio [HR] 1.34, 95\% confidence interval [CI] 0.93-1.91, $P=0.14$ ), or overall survival (HR 1.19, 95\% CI 0.73-1.93, $P=0.51$ ). Pregnancy was not a factor in recurrence risk or survival on multivariate analysis. ${ }^{35}$

\section{Termination of pregnancy}

Studies of pregnant women diagnosed with breast cancer fail to show a survival benefit with termination of the pregnancy, and this is a personal choice to be made on a case-by-case basis. ${ }^{25,36-40}$ Some studies showed an improved survival for patients who continued their pregnancies compared to those who terminated, but the bias of lower risk patients continuing versus those with more active disease choosing to terminate cannot be excluded as stage is not reported in the publications.

\section{Treatment}

The goals of breast cancer treatment are the same for pregnant and nonpregnant women: to control the cancer locally and prevent systemic spread. Systemic treatment includes adjuvant or neoadjuvant chemotherapy regardless of patient's age or stage at diagnosis. For patients with large tumors, neoadjuvant chemotherapy is chosen to identify a pathologic complete response, identify who will do well postoperatively, or to downstage a tumor and allow breast conservation, if desired. For patients diagnosed prior to 12 weeks when chemotherapy would not be an option, surgery should be performed, followed by adjuvant chemotherapy after 12 weeks of gestation. The treatment course may need to be altered due to pregnancy, ie, a patient diagnosed during the first trimester of pregnancy should undergo surgery only with chemotherapy delayed until the second trimester, even if the preference would have been to use neoadjuvant chemotherapy were she not pregnant.

Beadle et al closely evaluated the survival of 668 patients younger than 35 years of age with breast cancer: 51 diagnosed during pregnancy, 53 within 1 year postpartum, and 548 nonpregnant cases. During the median follow-up of 114 months, patients with pregnancyassociated cases had no statistically significant differences in 10-year locoregional recurrence, distant metastases, or overall survival. For patients diagnosed with breast cancer during pregnancy, any treatment intervention during pregnancy provided a trend toward improved overall survival compared to delaying evaluation and treatment until after delivery, $78.8 \%$ versus $44.6 \%, P=0.68 .{ }^{41}$ Loibl, too, found that delaying treatment until after delivery did not afford a survival advantage. ${ }^{23}$

In the majority of case series of systemic treatment during pregnancy, anthracyclines are most commonly used with cyclophosphamide with or without 5-fluouracil, and taxanes are often postponed until the postpartum. The literature includes mostly retrospective case reports or series, with limited long-term follow-up on the neonate after in utero exposure. One of the few prospective cohort studies was performed by Berry et al who treated 23 pregnant patients with breast cancer with doxorubicin (50 $\left.\mathrm{mg} / \mathrm{m}^{2}\right)$, cyclophosphamide $\left(500 \mathrm{mg} / \mathrm{m}^{2}\right)$, and 5-fluouracil $\left(1,000 \mathrm{mg} / \mathrm{m}^{2}\right)$ for a median of four cycles. (See Neonatal outcomes section.). Patients tolerated the same doses of chemotherapy they would have received were they not pregnant. ${ }^{42}$ Rouzier et al compared the pathological response of 48 patients with PABC to non-PABC as a marker of chemosensitivity and found no difference. Pregnant women should be offered the same life-saving treatment, expecting the same response to treatment. ${ }^{43}$ The challenge is that advances in treatment in nonpregnant patients are slow to be adopted in the treatment protocols for pregnant women. 
Taxanes are widely used as standard first-line treatment for high-risk early-stage and advanced/metastatic breast cancer in nonpregnant women, resulting in a better response rate and longer time to progression than standard anthracycline-based regimens. ${ }^{44-47}$ Evidence regarding fetal tolerance to taxane is accumulating. ${ }^{48-57}$ In Amant et al's collaborative study, 169 women received taxane therapy during pregnancy, and the authors reported no increased malformations. ${ }^{35}$ In 2012, birth weight, gestational age at delivery, rate of growth restriction, congenital anomalies, and incidence of maternal and neonatal neutropenia were compared between 15 cases treated with taxanes during pregnancy and 123 patients with breast or ovarian cancer not exposed to taxanes during pregnancy. There were no statistically significant differences between the two groups. ${ }^{48}$ Rouzier et al found a better response when taxanes were added to anthracycline-based regimens during pregnancy. ${ }^{43}$ If anthracycline-based chemotherapy is completed too early for a safe delivery, it may be considered to treat with taxane rather than delaying until the postpartum.

Dose-dense therapy, favored in nonpregnant patients with advanced disease, is infrequently used during pregnancy but not contraindicated. In a retrospective cohort study, 10 women treated with dose-dense chemotherapy every 2 weeks, were compared with 99 women who received conventional chemotherapy, with at least 3-week intervals. Birth weight, gestational age at delivery, rate of growth restriction, congenital anomalies, and incidence of maternal and neonatal neutropenia were not statistically different between the two groups ${ }^{58}$ Pregnant patients undergoing dose-dense chemotherapy who experience neutropenia can be safely treated with pegfilgrastim, if necessary. One hundred seventy-six pregnant women who received chemotherapy were identified from the Cancer and Pregnancy Registry at Cooper Medical School of Rowan University. Birth outcomes, white blood count at birth, and pediatric health were compared between the groups, one receiving pegfilgrastim (exposed) and a control group (unexposed) (ie, chemotherapy without pegfilgrastim). There was no statistically significant difference in gestational age at birth, or congenital anomalies, birth weight, incidence of long-term medical issues, mean white blood count, or neutropenia at birth between the newborns exposed to pegfilgrastim with chemotherapy and newborns exposed to chemotherapy alone. ${ }^{59}$

Pregnant women tolerate chemotherapy with less nausea and vomiting compared to when they require the same regimens postpartum. This raises the suspicion about the pharmacokinetic changes inherent during pregnancy which may affect free-drug levels. These include expanded plasma volume, increased glomerular filtration rate, and increased activity of liver enzymes. At the current time, it is recommended that chemotherapy doses during pregnancy be based on height and actual weight, not ideal or prepregnancy maternal weight. ${ }^{3}$ Antiemetics are safe to use during pregnancy.

The majority of breast cancers diagnosed during pregnancy are ER negative, similar to nonpregnant premenopausal women of similar age at diagnosis. In the less common case of ER positive pregnancy-associated cancers, the use of tamoxifen or other antiestrogens including aromatase inhibitors is not recommended during pregnancy. Trastuzumab is also contraindicated during pregnancy due to reports of reversible fetal oligohydramnios associated with its use. ${ }^{60-70}$ If a patient conceives while taking trastuzumab, this can be discontinued and the ongoing pregnancy maintained. ${ }^{71}$

Radiation for breast cancer is delayed until after delivery. Fetal radiation exposure would be unacceptably high at the standard dose of radiation for breast cancer. Fetal exposure can range from 3.9-15 rad during the first trimester, when the uterine fundus is farthest from the breast, to up to $200 \mathrm{rad}$ toward the end of pregnancy. ${ }^{72}$

\section{Neonatal outcomes}

In discussion of neonatal outcomes after treatment for maternal cancer during pregnancy, one must account for the gestational age at delivery, as preterm infants will incur more medical issues and long-term consequences compared to infants delivered closer to term. Another concern in regard to fetal exposure includes that of fetal cardiotoxicity secondary to anthracyclines, as this is a known risk of anthracyclines in adults and children. Amant et al performed postnatal echocardiograms on children exposed to anthracyclines during the second and third trimesters and found no harmful fetal cardiac effects after in utero exposure to doxorubicin. ${ }^{35}$ The authors identified a significant difference in ejection fraction and fractional shortening between patients and age- and sex-matched controls, but values in all exposed patients remained in the normal range. Additionally, cardiac dimensions, wall thickness, and left ventricular mass index were all within normal limits. ${ }^{35}$ Avilés et al conducted echocardiograms on 81 children after in utero exposure to anthracyclines. The children were followed with serial echocardiograms from birth to 29 years of age and demonstrated no abnormalities in cardiac function. ${ }^{73}$

When chemotherapy is warranted during pregnancy, treatment is not started before completing the first trimester, as this is the period of fetal organogenesis. As stated previously, 
publications detailing chemotherapy exposures during pregnancy, including some details about the appearance of the child at birth, are mostly retrospective case reports or series, and data on neonatal outcomes beyond the first year are limited. Described in the series by Berry et al, 23 patients diagnosed with breast cancer were treated with 5-fluoruracil, doxorubicin, and cyclophosphamide chemotherapy during the second and third trimesters at comparable doses to that given to nonpregnant women. No congenital anomalies occurred. One infant born just 2 days after exposure had transient leukopenia after a spontaneous preterm delivery. No infectious consequences occurred. ${ }^{42}$ (Premature infants often have leukopenia due to increased regulatory T-cell values and diminished interleukin 7 , so the effect may not have been secondary to chemotherapy exposure). Follow up on these children was reported by Hahn et al, who expanded this series to 57 women and surveyed parents/guardians about their children's health and development. Except for two children with educational needs and one with Down syndrome, all were developmentally appropriate, and all were reported to be healthy. ${ }^{74}$ Avilés et al reported long-term follow-up on 82 children exposed to chemotherapy for maternal hematological cancers diagnosed during pregnancy. Each underwent extensive developmental and neurological assessments with reassuring results. ${ }^{75,76}$ Amant et al performed developmental testing on 70 children exposed in utero to chemotherapy for various cancers, including those of 35 women treated for breast cancer and six exposed to taxanes. Children underwent clinical neurologic examinations and assessments of their general level of cognitive functioning (Bayley Scale of Infant Development or intelligence quotient test). The majority of children showed normal neurologic development after exposure to chemotherapy in utero. The children who tested below normal in neurodevelopment were concentrated in the group of children who delivered preterm. ${ }^{35}$

\section{Delivery and lactation}

Iatrogenic preterm deliveries are discouraged, as these can result in long-term health consequences for the neonate. A minimum of 3 weeks is suggested between a chemotherapy session and delivery to avoid neonatal immunosuppression. Should it occur, it is most often transient without infectious consequences, but this remains a risk in a neonatal unit for a neutropenic infant. Three weeks allows the placenta to metabolize the chemotherapy and allows excretion from the fetus. This will not be completely accomplished if delivery occurs close to the latest chemotherapy treatment, and for premature infants, there is a limited capacity compared to term infants to take over the metabolism of these drugs postnatally. To avoid this possible consequence, administering chemotherapy beyond 35 weeks is discouraged, as spontaneous labor is more likely any time after 37 weeks gestation. ${ }^{23}$

Iatrogenic deliveries indicated prior to term should be limited to late preterm deliveries between 35-37 weeks. This may be indicated for a patient who completes chemotherapy by 32-33 weeks in order to restart chemotherapy postpartum or for radiation therapy to begin. Patients on a schedule that lends to chemotherapy up to the 34 th week can be induced after 37 weeks if postpartum treatment is warranted. Cesarean section should be reserved for routine obstetrical indications. The spontaneous preterm delivery rate is not statistically different from the background population. As long as there are no signs of postpartum infection, chemotherapy can be resumed 7 days after delivery.

For women who start or continue with chemotherapy after delivery, breastfeeding is not recommended. Transient neutropenia in a breastfed infant was reported when the mother was undergoing treatment with cyclophosphamide. ${ }^{77,78}$ For women who have completed chemotherapy with substantial time before delivery to allow metabolism of the drug and any active metabolites, breastfeeding should not be contraindicated from the neonatal perspective. If breast surgery is planned soon after delivery, breastfeeding may be discouraged to avoid engorgement prior to surgery. Breastfeeding is not contraindicated for women undergoing radiotherapy; however, the infant should only be fed from the untreated breast because mastitis in the radiated breast is very difficult to treat.

\section{Conclusion}

The evaluation of a palpable mass found during pregnancy should be pursued as if the patient is not pregnant, excluding performing ultrasound as the primary modality before mammography, unlike in the nonpregnant state in which both are performed simultaneously or mammography is performed first. Biopsy and surgery should proceed as if the patient is not pregnant, with some modifications due to physiologic changes during pregnancy. Reconstruction is delayed until postpartum. Although some articles suggest pregnancy carries a worse prognosis, not entirely due to delays in diagnosis, termination of the pregnancy has not been shown to afford a survival benefit. The chemotherapy agents used and the timing of treatment will depend on the gestational age at diagnosis, with the plan to complete or discontinue chemotherapy by 34 weeks, allowing 3 weeks 
prior to delivery. Iatrogenic premature deliveries prior to 35 weeks are discouraged. Neonatal outcomes appear favorable after second and third trimester chemotherapy, including after taxane exposure.

\section{Acknowledgment}

The author would like to acknowledge Gunda Simpkins, RN, MSN, MPH for her help in the preparation, critical reading, and editing of this manuscript.

\section{Disclosure}

The author reports no conflicts of interest in this work.

\section{References}

1. Ahn BY, Kim HH, Moon WK, et al. Pregnancy- and lactation-associated breast cancer: mammographic and sonographic findings. J Ultrasound Med. 2003;22(5):491-497; quiz 498.

2. Wojcinski S, Stefanidou N, Hillemanns P, Degenhardt F. The biology of malignant breast tumors has an impact on the presentation in ultrasound: an analysis of 315 cases. BMC Womens Health. 2013;13:47.

3. Amant F, Deckers S, Van Calsteren K, et al. Breast cancer in pregnancy: recommendations of an international consensus meeting. Eur J Cancer. 2010;46(18):3158-3168.

4. Pavlidis N, Pentheroudakis G. The pregnant mother with breast cancer: diagnostic and therapeutic management. Cancer Treat Rev. 2005;31(6):439-447.

5. Woo JC, Yu T, Hurd TC. Breast cancer in pregnancy: a literature review. Arch Surg. 2003;138(1):91-98; discussion 99.

6. Ishida T, Yokoe T, Kasumi F, et al. Clinicopathologic characteristics and prognosis of breast cancer patients associated with pregnancy and lactation: analysis of case-control study in Japan. Jpn J Cancer Res. 1992;83(11):1143-1149.

7. Nettleton J, Long J, Kuhan D, Wu R, Shaeffer J, El-Mahdi A. Breast cancer during pregnancy: quantifying the risk of treatment delay. Obstet Gynecol. 1996;87(3):414-418.

8. Baker J, Ali A, Groch MW, Fordham E, Economou SG. Bone scanning in pregnant patients with breast carcinoma. Clin Nucl Med. 1987;12(7):519-524.

9. Duncan PG, Pope WD, Cohen MM, Greer N. Fetal risk of anesthesia and surgery during pregnancy. Anesthesiology. 1986;64(6):790-794.

10. Mazze RI, Källén B. Reproductive outcome after anesthesia and operation during pregnancy: a registry study of 5405 cases. Am J Obstet Gynecol. 1989;161(5):1178-1185.

11. Kuerer HM, Gwyn K, Ames FC, Theriault RL. Conservative surgery and chemotherapy for breast carcinoma during pregnancy. Surgery. 2002;131(1):108-110.

12. Rodriguez AO, Chew H, Cress R, et al. Evidence of poorer survival in pregnancy-associated breast cancer. Obstet Gynecol. 2008;112(1): 71-78.

13. Gentilini O, Cremonesi M, Trifirò G, et al. Safety of sentinel node biopsy in pregnant patients with breast cancer. Ann Oncol. 2004;15(9): 1348-1351.

14. Gentilini O, Cremonesi M, Toesca A, et al. Sentinel lymph node biopsy in pregnant patients with breast cancer. Eur J Nucl Med Mol Imaging. 2010;37(1):78-83.

15. Pandit-Taskar N, Dauer LT, Montgomery L, St Germain J, Zanzonico PB, Divgi CR. Organ and fetal absorbed dose estimates from $99 \mathrm{mTc}$-sulfur colloid lymphoscintigraphy and sentinel node localization in breast cancer patients. J Nucl Med. 2006;47(7):1202-1208.

16. Keleher A, Wendt R, Delpassand E, Stachowiak AM, Kuerer HM. The safety of lymphatic mapping in pregnant breast cancer patients using Tc-99m sulfur colloid. Breast J. 2004;10(6):492-495.
17. Morita ET, Chang J, Leong SP. Principles and controversies in lymphoscintigraphy with emphasis on breast cancer. Surg Clin North Am. 2000;80(6):1721-1739.

18. Bonnier P, Romain S, Dilhuydy JM, et al. Influence of pregnancy on the outcome of breast cancer: a case-control study. Societe Francaise de Senologie et de Pathologie Mammaire Study Group. Int J Cancer. 1997;72(5):720-727.

19. Shousha S. Breast carcinoma presenting during or shortly after pregnancy and lactation. Arch Pathol Lab Med. 2000;124(7): 1053-1060.

20. Middleton LP, Amin M, Gwyn K, Theriault R, Sahin A. Breast carcinoma in pregnant women: assessment of clinicopathologic and immunohistochemical features. Cancer. 2003;98(5):1055-1060.

21. Reed W, Sandstad B, Holm R, Nesland JM. The prognostic impact of hormone receptors and c-erbB-2 in pregnancy-associated breast cancer and their correlation with BRCA1 and cell cycle modulators. Int J Surg Pathol. 2003;11(2):65-74.

22. Elledge RM, Ciocca DR, Langone G, McGuire WL. Estrogen receptor, progesterone receptor, and HER-2/neu protein in breast cancers from pregnant patients. Cancer. 1993;71(8):2499-2506.

23. Loibl S, von Minckwitz G, Gwyn K, et al. Breast carcinoma during pregnancy. International recommendations from an expert meeting. Cancer. 2006;106(2):237-246.

24. Petrek JA, Dukoff R, Rogatko A. Prognosis of pregnancy-associated breast cancer. Cancer. 1991;67(4):869-872.

25. Zemlickis D, Lishner M, Degendorfer P, et al. Maternal and fetal outcomes after breast cancer in pregnancy. Am J Obstet Gynecol. 1992;166(3):781-787.

26. Halaska MJ, Pentheroudakis G, Strnad P, et al. Presentation, management and outcome of 32 patients with pregnancy-associated breast cancer: a matched controlled study. Breast J. 2009;15(5):461-467.

27. Ibrahim EM, Ezzat AA, Baloush A, Hussain ZH, Mohammed GH. Pregnancy-associated breast cancer: a case-control study in a young population with a high-fertility rate. Med Oncol. 2000;17(4): 293-300.

28. Zhang J, Liu G, Wu J, et al. Pregnancy-associated breast cancer: a case control and long-term follow-up study in China. J Exp Clin Cancer Res. 2003;22(1):23-27.

29. Smith LH, Danielsen B, Allen ME, Cress R. Cancer associated with obstetric delivery: results of linkage with the California cancer registry. Am J Obstet Gynecol. 2003;189(4):1128-1135.

30. Nugent P, O'Connell TX. Breast cancer and pregnancy. Arch Surg. 1985;120(11):1221-1224.

31. Azim HA, Santoro L, Russell-Edu W, Pentheroudakis G, Pavlidis N, Peccatori FA. Prognosis of pregnancy-associated breast cancer: a metaanalysis of 30 studies. Cancer Treat Rev. 2012;38(7):834-842.

32. Johansson AL, Andersson TM, Hsieh CC, et al. Stage at diagnosis and mortality in women with pregnancy-associated breast cancer (PABC). Breast Cancer Res Treat. 2013;139(1):183-192.

33. Anderson BO, Petrek JA, Byrd DR, Senie RT, Borgen PI. Pregnancy influences breast cancer stage at diagnosis in women 30 years of age and younger. Ann Surg Oncol. 1996;3(2):204-211.

34. Tretli S, Kvalheim G, Thoresen S, Høst H. Survival of breast cancer patients diagnosed during pregnancy or lactation. $\mathrm{Br} J$ Cancer. 1988;58(3):382-384.

35. Amant F, von Minckwitz G, Han SN, et al. Prognosis of women with primary breast cancer diagnosed during pregnancy: results from an international collaborative study. J Clin Oncol. 2013;31(20):2532-2539.

36. Psyrri A, Burtness B. Pregnancy-associated breast cancer. Cancer J. 2005;11(2):83-95.

37. Holleb AI, Farrow JH. The relation of carcinoma of the breast and pregnancy in 283 patients. Surg Gynecol Obstet. 1962;115:65-71

38. King RM, Welch JS, Martin JK Jr, Coulam CB. Carcinoma of the breast associated with pregnancy. Surg Gynecol Obstet. 1985;160(3): 228-232.

39. Clark RM, Chua T. Breast cancer and pregnancy: the ultimate challenge. Clin Oncol (R Coll Radiol). 1989;1(1):11-18. 
40. Jacobs IA, Chang CK, Salti GI. Coexistence of pregnancy and cancer. Am Surg. 2004;70(11):1025-1029.

41. Beadle BM, Woodward WA, Middleton LP, et al. The impact of pregnancy on breast cancer outcomes in women. Cancer. 2009;115(6): 1174-1184.

42. Berry DL, Theriault RL, Holmes FA, et al. Management of breast cancer during pregnancy using a standardized protocol. J Clin Oncol. 1999;17(3):855-861.

43. Rouzier R, Werkoff G, Uzan C, et al. Pregnancy-associated breast cancer is as chemosensitive as non-pregnancy-associated breast cancer in the neoadjuvant setting. Ann Oncol. 2011;22(7):1582-1587.

44. De Laurentiis M, Cancello G, D'Agostino D, et al. Taxane-based combinations as adjuvant chemotherapy of early breast cancer: a metaanalysis of randomized trials. J Clin Oncol. 2008;26(1):44-53.

45. Martin M, Pienkowski T, Mackey J, et al; Breast Cancer International Research Group 001 Investigators. Adjuvant docetaxel for node-positive breast cancer. $N$ Engl J Med. 2005;352(22):2302-2313.

46. Sparano JA, Wang M, Martino S, et al. Weekly paclitaxel in the adjuvant treatment of breast cancer. $N$ Engl J Med. 2008;358(16):1663-1671.

47. Jones S, Holmes FA, O'Shaughnessy J, et al. Docetaxel with Cyclophosphamide is Associated with an Overall Survival Benefit Compared with Doxorubicin and Cyclophosphamide: 7-Year Follow-up of US Oncology Research Trial 9735. J Clin Oncol. 2009;27(8): $1177-1183$.

48. Cardonick E, Bhat A, Gilmandyar D, Somer R. Maternal and fetal outcomes of taxane chemotherapy in breast and ovarian cancer during pregnancy: case series and review of the literature. Ann Oncol. 2012;23(12):3016-3023.

49. Sood AK, Shahin MS, Sorosky JI. Paclitaxel and platinum chemotherapy for ovarian carcinoma during pregnancy. Gynecol Oncol. 2001;83(3):599-600.

50. Méndez LE, Mueller A, Salom E, González-Quintero VH. Paclitaxel and carboplatin chemotherapy administered during pregnancy for advanced epithelial ovarian cancer. Obstet Gynecol. 2003; 102(5 Pt 2):1200-1202.

51. Rouzi AA, Sahly NN, Sahly NF, Alahwal MS. Cisplatinum and docetaxel for ovarian cancer in pregnancy. Arch Gynecol Obstet. 2009;280(5):823-825.

52. Chun KC, Kim DY, Kim JH, Kim YM, Kim YT, Nam JH. Neoadjuvant chemotherapy with paclitaxel plus platinum followed by radical surgery in early cervical cancer during pregnancy: three case reports. Jpn J Clin Oncol. 2010;40(7):694-698.

53. García-González J, Cueva J, Lamas MJ, Curiel T, Graña B, López-López R. Paclitaxel and cisplatin in the treatment of metastatic non-small-cell lung cancer during pregnancy. Clin Transl Oncol. 2008;10(6):375-376.

54. Kim JH, Kim HS, Sung CW, Kim KJ, Kim CH, Lee KY. Docetaxel, gemcitabine, and cisplatin administered for non-small cell lung cancer during the first and second trimester of an unrecognized pregnancy. Lung Cancer. 2008;59(2):270-273.

55. Azim HA, Scarfone G, Peccatori FA. Carboplatin and weekly paclitaxel for the treatment of advanced non-small cell lung cancer (NSCLC) during pregnancy. J Thorac Oncol. 2009;4(4):559-560.

56. Mir O, Berveiller $P$, Goffinet $F$, et al. Taxanes for breast cancer during pregnancy: a systematic review. Ann Oncol. 2010;21(2):425-426.

57. Serkies K, Węgrzynowicz E, Jassem J. Paclitaxel and cisplatin chemotherapy for ovarian cancer during pregnancy: case report and review of the literature. Arch Gynecol Obstet. 2011;283(Suppl 1):97-100.

58. Cardonick E, Gilmandyar D, Somer RA. Maternal and neonatal outcomes of dose-dense chemotherapy for breast cancer in pregnancy. Obstet Gynecol. 2012;120(6):1267-1272.
59. Cardonick E, Irfan F, Torres N. The use of neupogen (filgrastim) or neulasta (pegfilgrastim) during pregnancy when chemotherapy is indicated for maternal cancer treatment. $J$ Cancer Ther. 2012;3(2):157-161.

60. Witzel ID, Müller V, Harps E, Janicke F, Dewit M. Trastuzumab in pregnancy associated with poor fetal outcome. Ann Oncol. 2008;19(1): 191-192.

61. Sekar R, Stone PR. Trastuzumab use for metastatic breast cancer in pregnancy. Obstet Gynecol. 2007;110(2 Pt 2):507-510.

62. Shrim A, Garcia-Bournissen F, Maxwell C, Farine D, Koren G. Favorable pregnancy outcome following Trastuzumab (Herceptin) use during pregnancy - case report and updated literature review. Reprod Toxicol. 2007;23(4):611-613.

63. Bader AA, Schlembach D, Tamussino KF, Pristauz G, Petru E. Anhydramnios associated with administration of trastuzumab and paclitaxel for metastatic breast cancer during pregnancy. Lancet Oncol. 2007;8(1):79-81.

64. Waterston AM, Graham J. Effect of adjuvant trastuzumab on pregnancy. J Clin Oncol. 2006;24(2):321-322.

65. Fanale MA, Uyei AR, Theriault RL, Adam K, Thompson RA. Treatment of metastatic breast cancer with trastuzumab and vinorelbine during pregnancy. Clin Breast Cancer. 2005;6(4):354-356.

66. Watson WJ. Herceptin (trastuzumab) therapy during pregnancy: association with reversible anhydramnios. Obstet Gynecol. 2005;105(3): 642-643.

67. Azim HA, Peccatori FA, Liptrott SJ, Catania C, Goldhirsch A. Breast cancer and pregnancy: how safe is trastuzumab? Nat Rev Clin Oncol. 2009;6(6):367-370.

68. Beale JM, Tuohy J, McDowell SJ. Herceptin (trastuzumab) therapy in a twin pregnancy with associated oligohydramnios. Am J Obstet Gynecol. 2009;201(1):e13-e14.

69. Weber-Schoendorfer C, Schaefer C. Trastuzumb exposure during pregnancy. Reprod Toxicol. 2008;25(3):390-391; author reply 392.

70. Pant S, Landon MB, Blumenfeld M, Farrar W, Shapiro CL. Treatment of breast cancer with trastuzumab during pregnancy. J Clin Oncol. 2008;26(9):1567-1569.

71. Zagouri F, Sergentanis TN, Chrysikos D, Papadimitriou CA, Dimopoulos MA, Bartsch R. Trastuzumab administration during pregnancy: a systematic review and meta-analysis. Breast Cancer Res Treat. 2013;137(2):349-357.

72. Antypas C, Sandilos P, Kouvaris J, et al. Fetal dose evaluation during breast cancer radiotherapy. Int J Radiat Oncol Biol Phys. 1998;40(4):995-999.

73. Avilés A, Neri N, Nambo MJ. Long-term evaluation of cardiac function in children who received anthracyclines during pregnancy. Ann Oncol. 2006;17(2):286-288.

74. Hahn KM, Johnson PH, Gordon N, et al. Treatment of pregnant breast cancer patients and outcomes of children exposed to chemotherapy in utero. Cancer. 2006;107(6):1219-1226.

75. Avilés A, Díaz-Maqueo JC, Talavera A, Guzmán R, García EL. Growth and development of children of mothers treated with chemotherapy during pregnancy: current status of 43 children. Am J Hematol. 1991;36(4):243-248.

76. Avilés A, Neri N. Hematological malignancies and pregnancy: a final report of 84 children who received chemotherapy in utero. Clin Lymphoma. 2001;2(3):173-177.

77. Amato D, Niblett JS. Neutropenia from cyclophosphamide in breast milk. Med J Aust. 1977;1(11):383-384.

78. Durodola JI. Administration of cyclophosphamide during late pregnancy and early lactation: a case report. J Natl Med Assoc. 1979;71(2):165-166. 
International Journal of Women's Health

Dovepress

\section{Publish your work in this journal}

The International Journal of Women's Health is an international, peerreviewed open-access journal publishing original research, reports, editorials, reviews and commentaries on all aspects of women's healthcare including gynecology, obstetrics, and breast cancer. The manuscript management system is completely online and includes Visit http://www.dovepress.com/testimonials.php to read real quotes from published authors.

Submit your manuscript here: http://www.dovepress.com/international-journal-of-womens-health-journal 